\title{
An Improvement to Chvátal and Thomassen's Upper Bound for Oriented Diameter
}

\author{
Jasine Babu, Deepu Benson, Deepak Rajendraprasad and Sai Nishant Vaka \\ Indian Institute of Technology Palakkad
}

\begin{abstract}
An orientation of an undirected graph $G$ is an assignment of exactly one direction to each edge of $G$. The oriented diameter of a graph $G$ is the smallest diameter among all the orientations of $G$. The maximum oriented diameter of a family of graphs $\mathscr{F}$ is the maximum oriented diameter among all the graphs in $\mathscr{F}$. Chvátal and Thomassen [JCTB, 1978] gave a lower bound of $\frac{1}{2} d^{2}+d$ and an upper bound of $2 d^{2}+2 d$ for the maximum oriented diameter of the family of 2-edge connected graphs of diameter $d$. We improve this upper bound to $1.373 d^{2}+6.971 d-1$, which outperforms the former upper bound for all values of $d$ greater than or equal to 8 . For the family of 2-edge connected graphs of diameter 3, Kwok, Liu and West [JCTB, 2010] obtained improved lower and upper bounds of 9 and 11 respectively. For the family of 2 -edge connected graphs of diameter 4 , the bounds provided by Chvátal and Thomassen are 12 and 40 and no better bounds were known. By extending the method we used for diameter $d$ graphs, along with an asymmetric extension of a technique used by Chvátal and Thomassen, we have improved this upper bound to 21 .
\end{abstract}

Keywords - Oriented diameter, Strong orientation, One-way traffic problem

\section{Introduction}

An orientation of an undirected graph $G$ is an assignment of exactly one direction to each of the edges of $G$. A given undirected graph can be oriented in many different ways $\left(2^{m}\right.$, to be precise, where $m$ is the number of edges). The studies on graph orientations often concern with finding orientations which achieve a predefined objective. Some of the objectives while orienting graphs include minimization of certain distances, ensuring acyclicity, minimizing the maximum in-degree, maximizing connectivity, etc. One of the earliest studies regarding graph orientations were carried out by H.E. Robbins in 1939. He was trying to answer a question posed by Stanislaw Ulam. "When may the arcs of a graph be so oriented that one may pass from any vertex to any other, traversing arcs in the positive sense only?". This led to a seminal work [1] of Robbins in which he proved the following theorem, "A graph is orientable if and only if it remains connected after the removal of any arc".

A directed graph $\vec{G}$ is called strongly connected if it is possible to reach any vertex starting from any other vertex using a directed path. An undirected graph $G$ is called strongly orientable if it has a strongly connected orientation. A bridge in a connected graph is an edge whose removal will disconnect the graph. A 2-edge connected graph is a connected graph which does not contain any bridges. The theorem of Robbins stated earlier says that it is possible for a graph $G$ to be strongly oriented if and only if $G$ is 2-edge connected. Though Robbins stated the necessary and sufficient conditions for a graph to have a strong orientation, no comparison between the diameter of a graph and the diameter of an orientation of this graph was given in this study. This was taken up by Chvátal and Thomassen in 1978 [2].

In order to discuss these quantitative results, we introduce some notation. Let $G$ be an undirected graph. The distance between two vertices $u$ and $v$ of $G, d_{G}(u, v)$ is the number of edges in a shortest path between $u$ and $v$. For any two subsets $A, B$ of $V(G)$, let $d_{G}(A, B)=\min \left\{d_{G}(u, v): u \in A, v \in B\right\}$. The eccentricity of a vertex $v$ of $G$ is the maximum distance between $v$ and any other vertex $u$ of $G$. The diameter of $G$ is the maximum of the eccentricities of its vertices. The radius of $G$ is the minimum of the eccentricities of its vertices. Let $\vec{G}$ be a directed graph and $u, v \in V(\vec{G})$. Then the distance from a vertex $u$ to $v, d_{\vec{G}}(u, v)$, is defined as the length of a shortest 
directed path from $u$ to $v$. For any two subsets $A, B$ of $V(\vec{G})$, let $d_{\vec{G}}(A, B)=\min \left\{d_{\vec{G}}(u, v): u \in A, v \in B\right\}$. The out-eccentricity of a vertex $v$ of $\vec{G}$ is the greatest distance from $v$ to a vertex $u \in V(\vec{G})$. The in-eccentricity of a vertex $v$ of $\vec{G}$ is the greatest distance from a vertex $u \in V(\vec{G})$ to $v$. The eccentricity of a vertex $v$ of $\vec{G}$ is the maximum of its out-eccentricity and in-eccentricity. The diameter of $\vec{G}$, denoted by $d(\vec{G})$, is the maximum of the eccentricities of its vertices. The radius of $\vec{G}$ is the minimum of the eccentricities of its vertices. The oriented diameter of an undirected graph $G$, denoted by $\vec{d}(G)$, is the smallest diameter among all strong orientations of $G$. That is, $\vec{d}(G):=\min \{d(\vec{G}): \vec{G}$ is an orientation of $\mathrm{G}\}$. The oriented radius of an undirected graph $G$ is the smallest radius among all strong orientations of $G$. The maximum oriented diameter of the family $\mathscr{F}$ of graphs is the maximum oriented diameter among all the graphs in $\mathscr{F}$. Let $f(d)$ denote the maximum oriented diameter of the family of 2-edge connected diameter $d$ graphs. That is, $f(d):=\max \{\vec{d}(G): G \in \mathscr{F}\}$, where $\mathscr{F}$ is the family of 2-edge connected graphs with diameter $d$.

The following theorem by Chvátal and Thomassen [2] gives an upper bound for the oriented radius of a graph.

Theorem 1. [2] Every 2-edge connected graph of radius $r$ admits a strong orientation of radius at most $r^{2}+r$.

The above bound was also shown to be tight. In the same paper, they also proved that the problem of deciding whether an undirected graph admits an orientation of diameter 2 is NP-hard. Motivated by the work of Chvátal and Thomassen [2], Chung, Garey and Tarjan [3] proposed a linear-time algorithm to check whether a mixed multigraph has a strong orientation or not. They have also proposed a polynomial time algorithm which provides a strong orientation (if it exists) for a mixed multigraph with oriented radius at most $4 r^{2}+4 r$. Studies have also been carried out regarding the oriented diameter of specific subclasses of graphs like AT-free graphs, interval graphs, chordal graphs and planar graphs [4, 5,6]. Bounds on oriented diameter in terms of other graph parameters like minimum degree and maximum degree are also available in literature $[7,8,9,10]$.

The following bounds for $f(d)$ were given by Chvátal and Thomassen [2].

Theorem 2. [2] $\frac{1}{2} d^{2}+d \leq f(d) \leq 2 d^{2}+2 d$.

Chvátal and Thomassen [2] has also proved that $f(2)=6$. By Theorem 2, $8 \leq f(3) \leq 24$. In 2010, Kwok, Liu and West [11] improved these bounds to $9 \leq f(3) \leq 11$. To prove the upper bound of 11, Kwok, Liu and West partitioned the vertices of $G$ into a number of sets based on the distances from the endpoints of an edge which is not part of any 3-cycle. Our study on the oriented diameter of 2-edge connected graphs with diameter $d$ uses this idea of partitioning the vertex set into a number of sets based on their distances from a specific edge.

\section{Our Results}

In this paper we establish two improved upper bounds. Firstly in Section 2, we show that $f(d) \leq 1.373 d^{2}+$ $6.971 d-1$ (Theorem 7). This is the first general improvement to Chvátal and Thomassen's upper bound $f(d) \leq$ $2 d^{2}+2 d$ from 1978. For all $d \geq 8$, our upper bound outperforms that of Chvátal and Thomassen. Their lower bound $f(d) \geq \frac{1}{2} d^{2}+d$ still remains unimproved. We do not believe that our upper bound is tight. Secondly in Section 3, for the case of $d=4$, we further sharpen our analysis and show that $f(4) \leq 21$ (Theorem 13). This is a considerable improvement from 40, which follows from Chvátal and Thomassen's general upper bound. Here too, our upper bound is not yet close to the lower bound of 12 given by Chvátal and Thomassen and we believe that there is room for improvement in the upper bound.

\section{Oriented Diameter of Diameter $d$ Graphs}

A subset $D$ of the vertex set of $G$ is called a $k$-step dominating set of $G$ if every vertex not in $D$ is at a distance of at most $k$ from at least one vertex of $D$. An oriented subgraph $\vec{H}$ of $G$ is called a $k$-step dominating oriented subgraph if $V(\vec{H})$ is a $k$-step dominating set of $V(G)$. To obtain upper bounds for the oriented diameter of a graph $G$ with $n$ vertices and minimum degree $\delta \geq 2$, Bau and Dankelmann [7] and Surmacs [8] first constructed a 2-step dominating oriented subgraph $\vec{H}$ of $G$. They used this together with the idea in the proof of Theorem 1 on $\vec{H}$ to obtain the upper bounds of $\frac{11 n}{\delta+1}+9$ and $\frac{7 n}{\delta+1}$, respectively, for the oriented diameter of graphs with minimum degree $\delta \geq 2$. We are using the algorithm ORIENTEDCORE described below to produce a 2-edge connected oriented subgraph $\vec{H}$ of $G$ with some distance guarantees between the vertices in $\vec{H}$ (Lemma 3 ) and some domination properties (Lemma 5). 


\subsection{Algorithm OrientedCore}

Input: A 2-edge connected graph $G$ and a specified edge $p q$ in $G$.

Output: A 2-edge connected oriented subgraph $\vec{H}$ of $G$.

Terminology: Let $d$ be the diameter of $G$, let $k$ be the length of a smallest cycle containing $p q$ in $G$ and let $h=\lfloor k / 2\rfloor$. Notice that $k \leq 2 d+1$ and $h \leq d$. Define $S_{i, j}=\left\{v \in V(G): d_{G}(v, p)=i, d_{G}(v, q)=j\right\}$. Since $S_{i, j}$ is non-empty only if $0 \leq i, j \leq d$ and $|i-j| \leq 1$, we implicitly assume these restrictions on the subscripts of $S_{i, j}$ wherever we use it. For a vertex $v \in S_{i, j}$, its level $L(v)$ is $(j-i)$ and its width $W(v)$ is $\max (i, j)$. We will always refer to an edge $\{u, v\}$ between two different $S_{i, j}$ 's as $u v$ when either $L(u)>L(v)$ or $L(u)=L(v)$ and $W(u)<W(v)$ (downward or rightward in Fig. 1). Moreover the edge $u v$ is called vertical in the first case and horizontal in the second.

Observations based on the first edge of shortest paths from a vertex $v$ to $p$ or $v$ to $q$ : Every vertex $v \in S_{i, i+1}$, $1 \leq i \leq d-1$, is incident to a horizontal edge $u v$ with $u \in S_{i-1, i}$. Every vertex $v \in S_{i+1, i}, 1 \leq i \leq d-1$, is incident to a horizontal edge $u v$ with $u \in S_{i, i-1}$. Every vertex $v \in S_{i, i}, 1 \leq i \leq d$, is incident either to a horizontal edge $u v$ with $u \in S_{i-1, i-1}$ or two vertical edges $u v$ and $v x$ with $u \in S_{i-1, i}$ and $x \in S_{i, i-1}$. Consequently for any $v$ in Level 1 , all the shortest $p-v$ path consists of Level 1 horizontal edges only and for any vertex in $v$ in Level 1 , all the shortest $v-q$ path consists of Level -1 horizontal edges alone. For any vertex $v$ in Level 0 , all the shortest $p-v$ path consists of horizontal edges in levels 1 and 0 and exactly one vertical edge; while all the shortest $v-q$ path consists of horizontal edges in levels 0 and -1 and exactly one vertical edge.

Stage 1. Initialise $\vec{H}$ to be empty. For each vertical edge $u v$ with $L(u)=1$ and $L(v) \in\{0,-1\}$, and for each shortest $p-u$ path $P_{u}$ and shortest $v-q$ path $P_{v}$, do the following: Let $P$ be the $p-q$ path formed by joining $P_{u}$, the edge $u v$ and $P_{v}$. Orient the path $P$ as a directed path $\vec{P}$ from $p$ to $q$ and add it to $\vec{H}$. Notice that even though two such paths can share edges, there is no conflict in the above orientation since, in Stage 1, every vertical edge is oriented downward, every horizontal edge in Level 1 is oriented rightward and every horizontal edge in levels 0 and -1 is oriented leftward.

Stage 2. For each vertical edge $u v$ with $L(u)=0$ and $L(v)=-1$ not already oriented in Stage 1 , and for each shortest $p-u$ path $P_{u}$ and shortest $v-q$ path $P_{v}$ do the following: Let $x$ be the last vertex in $P_{u}$ (nearest to $u$ ) that is already in $V(\vec{H})$ and let $P_{u}^{\prime}$ be the subpath of $P_{u}$ from $x$ to $u$. Similarly let $y$ be the first vertex in $P_{v}$ (nearest to $v$ ) that is already in $V(\vec{H})$ and let $P_{v}^{\prime}$ be the subpath of $P_{v}$ from $v$ to $y$. Let $P$ be the $x-y$ path formed by joining $P_{u}^{\prime}$, the edge $u v$ and $P_{v}^{\prime}$. Orient the path $P$ as a directed path $\vec{P}$ from $x$ to $y$ and add it to $\vec{H}$. Notice that $P$ does not share any edge with a path added to $\vec{H}$ in Stage 1 , but it can share edges with paths added in earlier steps of Stage 2. However there is no conflict in the orientation since, in Stage 2, every vertical edge is oriented downward, every horizontal edge in Level 0 is oriented rightward, every horizontal edge in Level -1 is oriented leftward, and no horizontal edges in Level 1 is added.

Stage 3. Finally orient the edge $p q$ from $q$ to $p$ and add it to $\vec{H}$. This completes the construction of $\vec{H}$, the output of the algorithm.

\section{Distances in $\vec{H}$}

First we analyse the (directed) distance from $p$ and to $q$ of vertices added to $\vec{H}$ in Stage 1 . The following bounds on distances in $\vec{H}$ follow from the construction of each path $P$ in Stage 1 . Let $w$ be any vertex that is added to $\vec{H}$ in Stage 1. Then

$$
\begin{aligned}
& d_{\vec{H}}(p, w) \leq \begin{cases}i, & w \in S_{i, i+1}, \\
h, & w \in S_{h, h}, \\
2 d-i, & w \in S_{i, i}, i>h, \text { and } \\
2 d-i, & w \in S_{i+1, i} .\end{cases} \\
& d_{\vec{H}}(w, q) \leq \begin{cases}2 d-i, & w \in S_{i, i+1}, \\
h, & w \in S_{h, h}, \\
2 d-i, & w \in S_{i, i}, i>h, \text { and } \\
i, & w \in S_{i+1, i} .\end{cases}
\end{aligned}
$$


It is easy to verify the above equations using the facts that $w$ is part of a directed $p-q$ path of length at most $2 d$ (at most $2 h$ if $w \in S_{h, h}$ ) in $\vec{H}$.

No new vertices from Level 1 or $S_{h, h}$ are added to $\vec{H}$ in Stage 2. Still the distance bounds for vertices added in Stage 2 are slightly more complicated since a path $P$ added in this stage will start from a vertex $x$ in Level 0 and end in a vertex $y$ in Level -1 , which are added to $\vec{H}$ in Stage 1 . But we can complete the analysis since we already know that $d_{\vec{H}}(p, x) \leq 2 d-h-1$ and $d_{\vec{H}}(y, q) \leq i$ where $i$ is such that $y \in S_{i+1, i}$ from the analysis of Stage 1 . Let $w$ be any vertex that is added to $\vec{H}$ in Stage 2 . Then

$$
d_{\vec{H}}(p, w) \leq\left\{\begin{array}{c}
(2 d-h-1)+(i-h-1) \\
=2 d-2 h-2+i, \quad w \in S_{i, i}, i>h, \text { and } \\
(2 d-h-1)+(d-h-1)+(d-i) \\
=4 d-2 h-2-i, \quad w \in S_{i+1, i} .
\end{array}\right.
$$

The distance from $w$ to $q$ in $\vec{H}$ is not affected even though we trim the path $P_{v}$ at $y$ since $y$ already has a directed shortest path to $q$ from Stage 1. Hence

$$
d_{\vec{H}}(w, q) \leq \begin{cases}2 d-i, & w \in S_{i, i}, i>h, \text { and } \\ i, & w \in S_{i+1, i} .\end{cases}
$$

The first part of the next lemma follows from taking the worst case among (1) and (3). Notice that $\forall i>$ $h,(2 h+2-i \leq i)$ and $(4 d-2 h-2 \geq 2 d)$ when $h<d$. New vertices are added to $\vec{H}$ in Stage 2 only if $h<d$. The second part follows from (2) and (4). The subsequent two claims are easy observations.

Lemma 3. Let $G$ be a 2-edge connected graph, pq be any edge of $G$ and let $\vec{H}$ be the oriented subgraph of $G$ returned by the algorithm ORIENTEDCORE. Then for every vertex $w \in V(\vec{H})$ we have

$$
\begin{gathered}
d_{\vec{H}}(p, w) \leq \begin{cases}i, & w \in S_{i, i+1}, \\
h, & w \in S_{h, h}, \\
2 d-2 h-2+i, & w \in S_{i, i}, i>h, \text { and } \\
4 d-2 h-2-i, & w \in S_{i+1, i} .\end{cases} \\
d_{\vec{H}}(w, q) \leq \begin{cases}2 d-i, & w \in S_{i, i+1}, \\
h, & w \in S_{h, h}, \\
2 d-i, & w \in S_{i, i}, i>h, \text { and } \\
i, & w \in S_{i+1, i} .\end{cases}
\end{gathered}
$$

Moreover, $d_{\vec{H}}(q, p)=1$ and $d_{\vec{H}}(p, q) \leq k-1$.

We can see that if $S_{h, h}$ is non-empty, then all the vertices in $S_{h, h}$ are captured into $\vec{H}$.

Notice that when $k \geq 4, S_{1,2}$ and $S_{2,1}$ are non empty. Thus the bound on the diameter of $\vec{H}$ follows by the triangle inequality $d_{\vec{H}}(x, y) \leq d_{\vec{H}}(x, q)+d_{\vec{H}}(q, p)+d_{\vec{H}}(p, y)$ and the fact that $\forall k \geq 4$ the worst bounds for $d_{\vec{H}}(x, q)$ and $d_{\vec{H}}(p, y)$ from Lemma 3 are when $x \in S_{1,2}$ and $y \in S_{2,1}$. Hence the following corollary.

Corollary 4. Let $G$ be a 2-edge connected graph, pq be any edge of $G$ and let $\vec{H}$ be the oriented subgraph of $G$ returned by the algorithm ORIENTEDCORE. If the length of the smallest cycle containing pq is greater than or equal to 4 , then the diameter of $\vec{H}$ is at most $6 d-2 h-3$.

\section{Domination by $\vec{H}$}

Let us call the vertices in $V(\vec{H})$ as captured and those in $V(G) \backslash V(\vec{H})$ as uncaptured. For each $i \in\{1,0,-1\}$ let $L_{i}^{c}$ and $L_{i}^{u}$ denote the captured and uncaptured vertices in level i, respectively. Since $L_{i}^{c}$ contains every level $i$ vertex incident with a vertical edge, $L_{i}^{c}$ separates $L_{i}^{u}$ from rest of $G$. Let $d_{i}$ denote the maximum distance between a vertex in $L_{i}^{u}$ and the set $L_{i}^{c}$. If $u_{i} \in L_{i}^{u}$ and $u_{j} \in L_{j}^{u}$ such that $d_{G}\left(u_{i}, L_{i}^{c}\right)=d_{i}$ and $d_{G}\left(u_{j}, L_{j}^{c}\right)=d_{j}$ for distinct $i, j \in\{1,0,-1\}$, the distance $d_{G}\left(u_{i}, u_{j}\right)$ is bounded above by $d$, the diameter of $G$, and bounded below by $d_{i}+1+d_{j}$. Hence $d_{i}+d_{j} \leq d-1$ for every distinct $i, j \in\{1,0,-1\}$. 
For any vertex $u \in L_{0}^{u}$, the last Level 0 vertex in a shortest (undirected) $u-q$ path is in $L_{0}^{c}$. Hence if Level 0 is non-empty then $d_{0} \leq(d-h)$. In order to bound $d_{1}$ and $d_{-1}$, we take a close look at a shortest cycle $C$ containing the edge $p q$. Let $C=\left(v_{1}, \ldots, v_{k}, v_{1}\right)$ with $v_{1}=q$ and $v_{k}=p$. Each $v_{i}$ is in $S_{i, i-1}$ when $2 i<k+1, S_{i-1, i-1}$ if $2 i=k+1$ and $S_{k-i, k-i+1}$ when $2 i>k+1$. Let $t=\lceil k / 4\rceil$. The Level -1 vertex $v_{t}$ is special since it is at a distance $t$ from Level 1 and thus $L_{1}^{c}$. If $u_{1}$ is a vertex in $L_{1}^{u}$ such that $d_{G}\left(u_{1}, L_{1}^{c}\right)=d_{1}$, the distance $d_{G}\left(u_{1}, v_{t}\right)$ is bounded above by $d$ and below by $d_{1}+t$. Hence $d_{1} \leq d-t$. Similarly we can see that $d_{-1} \leq(d-t)$.

Putting all these distance bounds on domination together, we get the next lemma.

Lemma 5. Let $G$ be a 2-edge connected graph, pq be any edge of $G$ and let $\vec{H}$ be the oriented subgraph of $G$ returned by the algorithm ORIENTEDCORE. For each $i \in\{1,0,-1\}$, let $d_{i}$ denote the maximum distance of a level $i$ vertex not in $V(\vec{H})$ to the set of level $i$ vertices in $V(\vec{H})$. Then $d_{0} \leq d-\lfloor k / 2\rfloor, d_{1}, d_{-1} \leq d-\lceil k / 4\rceil$ and for any distinct $i, j \in\{1,0,-1\}, d_{i}+d_{j} \leq d-1$.

\subsection{The Upper Bound}

Consider a 2-edge connected graph $G$ with diameter $d$. Let $\eta(G)$ denote the smallest integer such that every edge of a graph $G$ belongs to a cycle of length at most $\eta(G)$. Sun, Li, Li and Huang [12] proved the following theorem.

Theorem 6. [12] $\vec{d}(G) \leq 2 r(\eta-1)$ where $r$ is the radius of $G$ and $\eta=\eta(G)$.

We know that $r \leq d$ and hence we have $\vec{d}(G) \leq 2 d(\eta-1)$ as our first bound. Let $p q$ be an edge in $G$ such that the length of a smallest cycle containing $p q$ is $\eta$. If $\eta \leq 3$, then $\vec{d}(G) \leq 4 d$ which is smaller than the bound claimed in Theorem 7. So we assume $\eta \geq 4$. By Corollary $4, G$ has an oriented subgraph $\vec{H}$ with diameter at most $6 d-2\left\lfloor\frac{\eta}{2}\right\rfloor-3$. Moreover by Lemma $5, \vec{H}$ is a $\left(d-\left\lceil\frac{\eta}{4}\right\rceil\right)$-step dominating subgraph of $G$. Let $G_{0}$ be a graph obtained by contracting the vertices in $V(\vec{H})$ into a single vertex $v_{H}$. We can see that $G_{0}$ has radius at most $\left(d-\left\lceil\frac{\eta}{4}\right\rceil\right)$. Thus by Theorem $1, G_{0}$ has a strong orientation $\overrightarrow{G_{0}}$ with radius at most $\left(d-\left\lceil\frac{\eta}{4}\right\rceil\right)^{2}+\left(d-\left\lceil\frac{\eta}{4}\right\rceil\right)$. Since $d \leq 2 r$, we have $d\left(\overrightarrow{G_{0}}\right) \leq 2\left(d-\left\lceil\frac{\eta}{4}\right\rceil\right)^{2}+2\left(d-\left\lceil\frac{\eta}{4}\right\rceil\right)$. Notice that $\vec{G}_{0}$ and $\vec{H}$ do not have any common edges. Hence $G$ has an orientation with diameter at most $2\left(d-\left\lceil\frac{\eta}{4}\right\rceil\right)^{2}+2\left(d-\left\lceil\frac{\eta}{4}\right\rceil\right)+\left(6 d-2\left\lfloor\frac{\eta}{2}\right\rfloor-3\right)$ by combining the orientations in $\vec{H}$ and $\vec{G}_{0}$. Let $\eta=4 \alpha d$. Hence we get $\vec{d}(G) \leq \min \left\{8 \alpha d^{2}-2 d, 2(1-\alpha)^{2} d^{2}+8 d-6 \alpha d-1\right\}$. We can see that the dominant term in the first bound is $8 \alpha d^{2}$ while the dominant term in the second bound is at most $2(1-\alpha)^{2} d^{2}$. Notice that $0<\frac{3}{4 d} \leq \alpha \leq \frac{2 d+1}{4 d}<1$. Thus by optimizing for $\alpha$ in the range $(0,1)$, we obtain the following theorem.

Theorem 7. $f(d) \leq 1.373 d^{2}+6.971 d-1$.

For any $d \geq 8$, the above upper bound is an improvement over the upper bound of $2 d^{2}+2 d$ provided by Chvátal and Thomassen.

\section{Oriented Diameter of Diameter 4 Graphs}

Throughout this section, we consider $G$ to be an arbitrary 2-edge connected diameter 4 graph. We will show that the oriented diameter of $G$ is at most 21 and hence $f(4) \leq 21$. The following lemma by Chvátal and Thomassen [2] is used when $\eta(G) \leq 4$.

Lemma 8. [2] Let $\Gamma$ be a 2-edge connected graph. If every edge of $\Gamma$ lies in a cycle of length at most $k$, then it has an orientation $\vec{\Gamma}$ such that

$$
d_{\vec{\Gamma}}(u, v) \leq\left((k-2) 2^{\lfloor(k-1) / 2\rfloor}+1\right) d_{\Gamma}(u, v) \quad \forall u, v \in V(\vec{\Gamma})
$$

Hence if all edges of the graph $G$ lie in a 3-cycle or a 4-cycle, the oriented diameter of $G$ will be at most 20. Hence we can assume the existence of an edge $p q$ which is not part of any 3-cycle or 4-cycle as long as we are trying to prove an upper bound of 20 or more for $f(4)$. We apply algorithm ORIENTDCORE on $G$ with the edge $p q$ to obtain an oriented subgraph $\vec{H}_{1}$ of $G$. Fig. 1 shows a coarse representation of $\vec{H}_{1}$. 


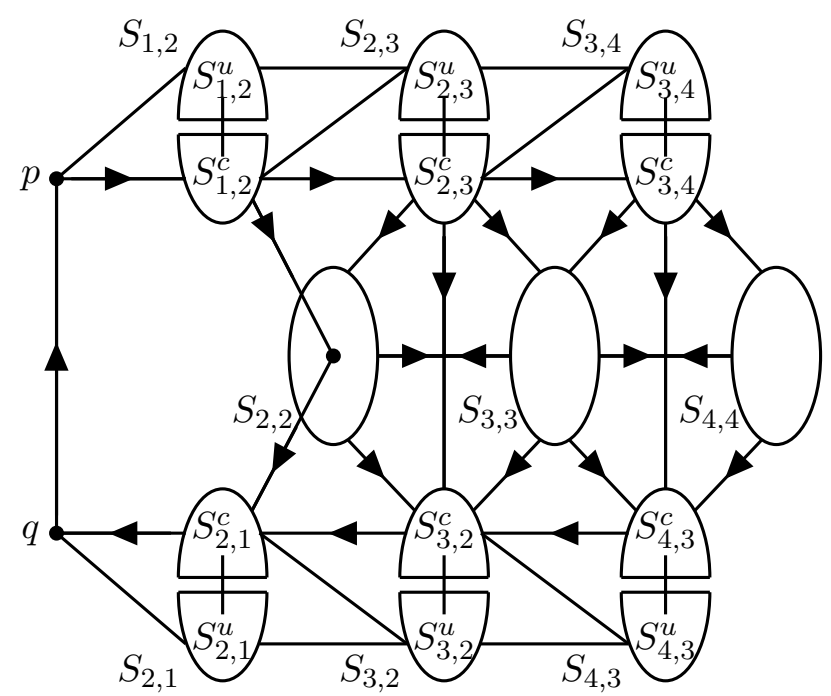

Figure 1: A coarse representation of $\vec{H}_{1}$ which shows the orientation of edges between various subsets of $V(G)$. A single arrow from one part to another indicates that all the edges between these parts are oriented from the former to latter. A double arrow between two parts indicates that the edges between the two parts are oriented in either direction or unoriented. An unoriented edge between two parts indicate that no edge between these two parts are oriented.

\begin{tabular}{|c||c|c|c|c|c|c|c|c|c|}
\hline for $w$ in & $S_{12}^{c}$ & $S_{23}^{c}$ & $S_{34}^{c}$ & $S_{22}$ & $S_{33}^{c}$ & $S_{44}^{c}$ & $S_{21}^{c}$ & $S_{32}^{c}$ & $S_{43}^{c}$ \\
\hline$d_{\vec{H}_{1}}(p, w) \leq$ & 1 & 2 & 3 & 2 & 5 & 6 & 9 & 8 & 7 \\
\hline$d_{\vec{H}_{1}}(w, q) \leq$ & 7 & 6 & 5 & 2 & 5 & 4 & 1 & 2 & 3 \\
\hline
\end{tabular}

Table 1: Upper bounds on the distances of $\vec{H}_{1}$

\subsection{Oriented Diameter and 2-Step Domination Property of $\vec{H}_{1}$}

Let $\vec{H}_{1}$ be the oriented subgraph of $G$ returned by the algorithm ORIENTEDCORE. Since the smallest cycle containing $p q$ is of length greater than or equal to 5 , by Corollary 4 , we can see that the diameter of $\vec{H}_{1}$ is at most 17. Moreover, from equations 5 and 6 of Lemma 3, we get the upper bounds on the distances of $\vec{H}_{1}$ in Table 1 . Hence, the following corollary.

Corollary 9. $d\left(\vec{H}_{1}\right) \leq 17$. Moreover $\forall w \in V\left(\vec{H}_{1}\right), d_{\vec{H}_{1}}(p, w)$ and $d_{\vec{H}_{1}}(w, q)$ obey the bounds in Table 1 .

Remark 1. If $k>5(h>2)$, then $S_{2,2}$ is empty. Moreover if $S_{2,2}$ is non-empty, then all the vertices in $S_{2,2}$ are captured into $\vec{H}_{1}$.

Furthermore, applying Lemma 5 on $\vec{H}_{1}$ shows that $\vec{H}_{1}$ is a 2-step dominating subgraph of $G$. Let $G_{0}$ be a graph obtained by contracting the vertices in $V\left(\vec{H}_{1}\right)$ into a single vertex $v_{H}$. We can see that $G_{0}$ has radius at most 2 . Thus by Theorem $1, G_{0}$ has a strong orientation $\overrightarrow{G_{0}}$ with radius at most 6 . Since $d \leq 2 r$, we have $d\left(\overrightarrow{G_{0}}\right) \leq 12$. Since $\vec{G}_{0}$ and $\vec{H}_{1}$ do not have any common edges we can see that $G$ has an orientation with diameter at most 29 by combining the orientations in $\overrightarrow{H_{1}}$ and $\overrightarrow{G_{0}}$. But we further improve this bound to 21 by constructing a 1 -step dominating oriented subgraph $\vec{H}_{2}$ of $G$. We propose the following asymmetric variant of a technique by Chvátal and Thomassen [2] for the construction and analysis of $\vec{H}_{2}$.

\subsection{Asymmetric Chvátal-Thomassen Lemma}

For any subset $A$ of $V(G)$, let $N(A)$ denote the set of all vertices with an edge incident on some vertex in $A$. Let $H$ be a subgraph of $G$. An ear of $H$ in $G$ is a sequence of edges $u v_{1}, v_{1} v_{2}, \ldots, v_{k-1} v_{k}, v_{k} v$ such that $u, v \in V(H)$, 
$k \geq 1$ and none of the vertices $v_{1}, \ldots, v_{k}$ and none of the edges in this sequence are in $H$. In particular we allow $u=v$.

Lemma 10 (Asymmetric Chvátal-Thomassen Lemma). Let $G$ be an undirected graph and let $A \subseteq B \subseteq V(G)$ such that

(i) $B$ is a k-step dominating set in $G$,

(ii) $G / B$ is 2-edge connected, and

(iii) $N(A) \cup B$ is a $(k-1)$-step dominating set of $G$.

Then there exists an oriented subgraph $\vec{H}$ of $G \backslash G[B]$ such that

(i) $N(A) \backslash B \subseteq V(\vec{H})$ and hence $V(\vec{H}) \cup B$ is a $(k-1)$-step dominating set of $G$, and

(ii) $\forall v \in V(\vec{H})$, we have $d_{\vec{H}}(A, v) \leq 2 k$ and either $d_{\vec{H}}(v, A) \leq 2 k$ or $d_{\vec{H}}(v, B \backslash A) \leq 2 k-1$.

Proof. We construct a sequence $\vec{H}_{0}, \vec{H}_{1}, \ldots$ of oriented subgraphs of $G \backslash G[B]$ as follows. We start with $\vec{H}_{0}=\emptyset$ and add an oriented $A-B$ ear $\vec{Q}_{i}$ in each step. Let $i \geq 0$. If $N(A) \backslash B \subseteq V\left(\vec{H}_{i}\right)$, then we stop the construction and set $\vec{H}=\vec{H}_{i}$. Since $N(A) \cup B$ is a $(k-1)$-step dominating set of $G$, the first conclusion of the lemma is satisfied when the construction ends with $N(A) \backslash B \subseteq V(\vec{H})$. If $N(A) \backslash B \nsubseteq \subseteq V\left(\vec{H}_{i}\right)$, then let $v \in(N(A) \backslash B) \backslash V\left(\vec{H}_{i}\right)$ and let $u$ be a neighbour of $v$ in $A$. Since $G / B$ is 2-edge connected, there exists a path in $G^{\prime}=(G / B) \backslash\{u v\}$ from $v$ to $B$. Let $P_{i}$ be a shortest $v-B$ path in $G^{\prime}$ with the additional property that once $P_{i}$ hits a vertex in an oriented ear $\vec{Q}_{j}$ that was added in a previous step, $P_{i}$ continues further to $B$ along the shorter arm of $Q_{j}$. It can be verified that $P_{i}$ is still a shortest $v-B$ path in $G^{\prime}$. The ear $Q_{i}$ is the union of the edge $u v$ and the path $P_{i}$. If $P_{i}$ hits $B$ without hitting any previous ear, then we orient $Q_{i}$ as a directed path $\vec{Q}_{i}$ from $u$ to $B$. If $Q_{i} \cap Q_{j} \neq \emptyset$, then we orient $Q_{i}$ as a directed path $\vec{Q}_{i}$ by extending the orientation of $Q_{i} \cap Q_{j}$. Notice that, in both these cases, the source vertex of $\vec{Q}_{i}$ is in $A$. We add $\vec{Q}_{i}$ to $\vec{H}_{i}$ to obtain $\vec{H}_{i+1}$.

Let $Q_{i}=\left(v_{0}, \ldots, v_{q}\right)$ with $v_{0} \in A$ and $v_{q} \in B$ be the ear added in the $i$-th stage above. Since $\left(v_{1}, \ldots, v_{q}\right)$ is a shortest $v_{1}-B$ path in $G^{\prime}=(G / B) \backslash\left\{v_{0} v_{1}\right\}$ and since $B$ is a $k$-step dominating set, $q \leq 2 k+1$. Moreover, if $v_{q} \in B \backslash A$, then $q \leq 2 k$ since $N(A) \cup B$ is a $(k-1)$-step dominating set. These bounds on the length of $Q_{i}$ along with the observation that the source vertex of $\vec{Q}_{i}$ is in $A$, verifies the second conclusion of the lemma.

Remark 2. If we flip the orientation of $\vec{H}$ we get the bounds $d_{\vec{H}}(v, A) \leq 2 k$ and either $d_{\vec{H}}(A, v) \leq 2 k$ or $d_{\vec{H}}(B \backslash A, v) \leq 2 k-1, \forall v \in V(\vec{H})$ in place of Conclusion (ii) of Lemma 10.

Setting $A=B$ in Lemma 10 gives the key idea which is recursively employed by Chvátal and Thomassen to prove Theorem 1 [2]. Notice from the above proof that, in this case $B \subseteq V(\vec{H})$. We can summarize their idea as follows.

Lemma 11 (Chvátal-Thomassen Lemma). Let $G$ be an undirected graph and let $B \subseteq V(G)$ such that

(i) $B$ is a $k$-step dominating set in $G$, and

(ii) $G / B$ is 2-edge connected.

Then there exists an oriented subgraph $\vec{H}$ of $G \backslash G[B]$ such that

(i) $V(\vec{H})$ is a $(k-1)$-step dominating set of $G$, and

(ii) $\forall v \in V(\vec{H})$, we have $d_{\vec{H}}(B, v) \leq 2 k$ and $d_{\vec{H}}(v, B) \leq 2 k$.

Let $G$ be any 2-edge connected graph with radius $r$. Chvátal and Thomassen showed that $\vec{d}(G) \leq 2 r+2(r-$ 1) $+\cdots+2=r(r+1)$ by $r$ applications of Lemma 11; starting with $B=\{v\}$, where $v$ is any central vertex of $G$ and $B$ in each subsequent application being the vertex-set of the oriented subgraph $\vec{H}$ returned by the current application. 


\subsection{A 1-Step Dominating Oriented Subgraph $\vec{H}_{2}$ of $G$}

Let $\vec{H}_{1}$ be the oriented subgraph of $G$ returned by the algorithm ORIENTEDCORE. We will add further oriented ears to $\vec{H}_{1}$ to obtain a 1-step dominating oriented subgraph $\vec{H}_{2}$ of $G$. We have already seen that $\vec{H}_{1}$ is a 2 -step dominating oriented subgraph of $G$. By Lemma 5, we also have $d_{i}+d_{j} \leq 3$ for any distinct $i, j \in\{1,0,-1\}$.

Now consider the first case where we have vertices in Level 0 which are at a distance 2 from $S_{2,2}$. Notice that in this case, $d_{0}=2$ and hence $d_{1}, d_{-1} \leq 1$. Let $B=L_{0}^{c}, A=S_{2,2}$ and $G_{0}=G\left[L_{0}\right]$. By Remark 1 , $A \subseteq B$. Notice that $B=L_{0}^{c}$ is a cut-set separating $L_{0}^{u}$ from the rest of $G$ and hence the graph $G_{0} / B$ is 2 edge connected. Since $S_{3,3}^{u} \subseteq N\left(S_{2,2}\right)$, we can see that $N(A) \cup B=N\left(S_{2,2}\right) \cup L_{0}^{c}$ is a 1-step dominating subgraph of $G_{0}$. Therefore we can apply Lemma 10 on $G_{0}$. Every edge of the oriented subgraph of $G_{0} \backslash G_{0}[B]$ obtained by applying Lemma 10 is reversed to obtain the subgraph $\overrightarrow{H_{2}^{0}}$. Now consider the vertices captured into $\overrightarrow{H_{2}^{0}}$. From Lemma 10 and Remark 2, we get the following bounds ${\overrightarrow{H_{2}^{0}}}_{2}(v, A) \leq 4$ and either $d_{\vec{H}_{2}^{0}}(A, v) \leq 4$ or $d_{\vec{H}_{2}^{0}}(B \backslash A, v) \leq 3, \forall v \in V\left(\overrightarrow{H_{2}^{0}}\right)$. Here $B \backslash A=S_{3,3}^{c} \cup S_{4,4}^{c}$ and from Table 1, we have the bounds $d_{\vec{H}_{1}}(p, x) \leq 5, \forall x \in S_{3,3}^{c}, d_{\vec{H}_{1}}(p, y) \leq 6, \forall y \in S_{4,4}^{c}$ and $d_{\vec{H}_{1}}(p, z)=2, \forall z \in S_{2,2}$. Hence $d_{\vec{H}_{1} \cup \vec{H}_{2}^{0}}(p, v) \leq 9$, $\forall v \in V\left(\overrightarrow{H_{2}^{0}}\right)$. Since $d_{\vec{H}_{2}^{0}}(v, A) \leq 4$ and $d_{\vec{H}_{1}}(x, q)=2, \forall x \in A$, we also have $d_{\vec{H}_{1} \cup \vec{H}_{2}^{0}}(v, q) \leq 6, \forall v \in V\left(\overrightarrow{H_{2}^{0}}\right)$. Let $\vec{H}_{2}=\vec{H}_{1} \cup \vec{H}_{2}^{0}$. By the above discussion, in combination with the distances in Table 1 and Corollary 9, we get the bounds in Table 2 for $d_{\vec{H}_{2}}(p, w)$ and $d_{\vec{H}_{2}}(w, q)$ when $V\left(\overrightarrow{H_{2}^{0}}\right) \neq \phi$. Moreover, $d\left(\vec{H}_{2}\right) \leq 17$.

\begin{tabular}{|c||c|c|c|c|c|c|c|c|c|}
\hline for $w$ in & $S_{12}^{c}$ & $S_{23}^{c}$ & $S_{34}^{c}$ & $S_{22}$ & $S_{33}^{c}$ & $S_{44}^{c}$ & $S_{21}^{c}$ & $S_{32}^{c}$ & $S_{43}^{c}$ \\
\hline$d_{\vec{H}_{2}}(p, w) \leq$ & 1 & 2 & 3 & 2 & $\mathbf{9}$ & $\mathbf{9}$ & 9 & 8 & 7 \\
\hline$d_{\vec{H}_{2}}(w, q) \leq$ & 7 & 6 & 5 & 2 & $\mathbf{6}$ & $\mathbf{6}$ & 1 & 2 & 3 \\
\hline
\end{tabular}

Table 2: Upper bounds on the distances of $\vec{H}_{2}$ when $V\left(\vec{H}_{2}^{0}\right) \neq \phi$

Now consider the second case where $d_{1}=2$ or $d_{-1}=2$. Since $d_{1}+d_{-1} \leq 3$, uncaptured vertices at a distance 2 from $H_{1}$ can exist either in Level 1 or in Level -1 but not both. By flipping the role of the vertices $p$ and $q$ in Algorithm ORIENTEDCORE if necessary, without loss of generality, we can assume vertices which are at a distance 2 from $\vec{H}_{1}$ exists only in Level -1 and not in Level 1 . Let $G_{-1}=G\left[L_{-1}\right]$ and $B=L_{-1}^{c}$. Further let $r$ be any vertex in $S_{1,2}^{c}$ and $A=\left\{v \in B: d_{G}(r, v)=2\right\}$. Since $q \in A, A$ is never empty. Note that $A \subseteq B \subseteq V\left(G_{-1}\right)$. Also $G_{-1} / B$ is 2-edge connected since $B=L_{-1}^{c}$ is a cut-set which separates $L_{-1}^{u}$ from the rest of $G$. Now consider a vertex $z$ in Level -1 which is exactly at a distance 2 from $B$. Since the graph $G$ is of diameter 4, there exists a 4-length path $P$ from $z$ to $r$. Since $B$ separates $L_{-1}^{u}$ from $r, P$ intersects $B$, say at a vertex $b$. Further, we have $d_{G}(b, r)=2$ and thus $b \in A$. Hence $z$ has a 2-length path to a vertex $b \in A$. Thus $N(A) \cup B$ is a 1-step dominating subgraph of $G_{-1}$. Hence we can apply Lemma 10 on $G_{-1}$ to obtain $\overrightarrow{H_{2}^{-1}}$, an oriented subgraph of $G_{-1} \backslash G_{-1}[B]$. Now consider the vertices captured into $\overrightarrow{H_{2}^{-1}}$. From Lemma 10, we get the following bounds $\forall v \in V\left(\overrightarrow{H_{2}^{-1}}\right), d_{H_{2}^{-1}}(A, v) \leq 4$ and $d_{H_{2}^{-1}}(v, B) \leq 4$. Since $d_{\vec{H}_{1}}(x, q) \leq 3, \forall x \in B$, we have $d_{\vec{H}_{1} \cup \vec{H}_{2}^{-1}}(v, q) \leq 7, \forall v \in V\left(\overrightarrow{H_{2}^{-1}}\right)$. Vertices in $A$ can be from $S_{2,1}^{c}, S_{3,2}^{c}$ or $\{q\}$. By the definition of $A$ there is an undirected path in $G$ of length 3 from $p$ to any vertex $v_{a}$ in $(A \backslash\{q\})$, going through $r$. It can be verified that this undirected path is oriented from $p$ to $v_{a}$ by Algorithm ORIENTEDCORE. Hence $d_{\vec{H}_{1}}\left(p, v_{a}\right) \leq 3, \forall v_{a} \in(A \backslash\{q\})$ and hence $\forall v \in V\left(\overrightarrow{H_{2}^{-1}}\right)$ with $d_{\vec{H}_{2}^{-1}}(A \backslash\{q\}, v) \leq 4, d_{\overrightarrow{H_{1}} \cup H_{2}^{-1}}(p, v) \leq 7$. But if a vertex $v \in V\left(\overrightarrow{H_{2}^{-1}}\right)$ has $d_{H_{2}^{-1}}(A \backslash\{q\}, v)>4$, then $d_{H_{2}^{-1}}^{\overrightarrow{H_{1}}}(q, v) \leq 4$. In this case, since $d_{\vec{H}_{1}}(p, q) \leq 8$, we get $d_{\overrightarrow{H_{1}} \cup \overrightarrow{H_{2}^{-}}}(p, v) \leq 12$. Notice that this is the only situation where $d_{\overrightarrow{H_{1}} \cup H_{2}^{-1}}(p, v)>9$ and in this particular case $d_{H_{2}^{-1}}(q, v) \leq 4$.

Now consider two vertices $x, y \in V\left(\vec{H}_{1} \cup{\overrightarrow{H_{2}^{-1}}}^{-1}\right.$. We can see that $d_{\vec{H}_{1} \cup H_{2}^{-1}}(x, y) \leq d_{\vec{H}_{1} \cup H_{2}^{-1}}(x, q)+$ $d_{\overrightarrow{H_{1}} \cup \vec{H}_{2}^{-1}}(q, y)$. We have already proved that $d_{\vec{H}_{1} \cup H_{2}^{-1}}(x, q) \leq 7$. Now let us consider the $q-y$ path. If $y \in V\left(\overrightarrow{H_{1}}\right)$, from Table 1 , we can see that $d_{\vec{H}_{1}}(p, y) \leq 9$ and therefore $d_{\overrightarrow{H_{1}} \cup \overrightarrow{H_{2}^{-1}}}(x, y) \leq 17$. Now suppose if $y \in\left(V\left(\overrightarrow{H_{2}^{-1}}\right) \backslash V\left(\overrightarrow{H_{1}}\right)\right)$. In this case we have already shown that $d_{H_{2}^{-1}}(p, y) \leq 9$ or $d_{H_{2}^{-1}}(q, y) \leq 4$. So, we either have a directed path of length 10 from $q$ to $y$ through $p$ or a directed path of length 4 to $y$ directly from $q$. 
Hence, $d_{\vec{H}_{1} \cup H_{2}^{-1}}(x, y) \leq 17$. Let ${\overrightarrow{H_{2}}}_{2}=\overrightarrow{H_{1}} \cup \overrightarrow{H_{2}^{-1}}$. By the above discussion, we get the bounds in Table 3 for $d_{\vec{H}_{2}}(p, w)$ and $d_{\overrightarrow{H_{2}}}(w, q)$ when $V\left(\overrightarrow{H_{2}^{-1}}\right) \neq \phi$. Moreover, $d\left(\overrightarrow{H_{2}}\right) \leq 17$.

\begin{tabular}{|c||c|c|c|c|c|c|c|c|c|}
\hline for $w$ in & $S_{12}^{c}$ & $S_{23}^{c}$ & $S_{34}^{c}$ & $S_{22}$ & $S_{33}^{c}$ & $S_{44}^{c}$ & $S_{21}^{c}$ & $S_{32}^{c}$ & $S_{43}^{c}$ \\
\hline$d_{\vec{H}_{2}}(p, w) \leq$ & 1 & 2 & 3 & 2 & 5 & 6 & $\mathbf{1 2}$ & $\mathbf{1 2}$ & $\mathbf{1 2}$ \\
\hline$d_{\vec{H}_{2}}(w, q) \leq$ & 7 & 6 & 5 & 2 & 5 & 4 & $\mathbf{7}$ & $\mathbf{7}$ & $\mathbf{7}$ \\
\hline
\end{tabular}

Table 3: Upper bounds on the distances of $\vec{H}_{2}$ when $V\left(\overrightarrow{H_{2}^{-1}}\right) \neq \phi$

In both the cases we get an oriented subgraph $\vec{H}_{2}$ of $G$ with $d\left(\vec{H}_{2}\right) \leq 17$. Moreover, it is clear from Conclusion (i) of Lemma 10 that $\vec{H}_{2}$ is a 1-step dominating subgraph of $G$. Hence the following Lemma.

Lemma 12. For every 2 -edge connected graph $G$ with diameter 4 and $\eta(G) \geq 5$, there exists a 1-step dominating oriented subgraph $\vec{H}_{2}$ of $G$ with $d\left(\vec{H}_{2}\right) \leq 17$.

\subsection{The Upper Bound}

Now the main theorem of the section follows.

Theorem 13. $f(4) \leq 21$.

Proof. By Lemma 12, we get a 1-step dominating oriented subgraph $\vec{H}_{2}$ of $G$ with $d\left(\vec{H}_{2}\right) \leq 17$. Let $G_{0}$ be a graph obtained by contracting the vertices in $V\left(\vec{H}_{2}\right)$ into a single vertex $v_{H}$. We can see that $G_{0}$ has radius at most 1. Thus by Theorem $1, G_{0}$ has a strong orientation $\overrightarrow{G_{0}}$ with radius at most 2 . Since $d \leq 2 r$, we have $d\left(\overrightarrow{G_{0}}\right) \leq 4$. Notice that $\vec{G}_{0}$ and $\vec{H}_{2}$ do not have any common edges. Now we can see that $G$ has an orientation with diameter at most 21 by combining the orientations in $\vec{H}_{2}$ and $\vec{G}_{0}$.

\section{References}

[1] Herbert Ellis Robbins. A theorem on graphs, with an application to a problem of traffic control. The American Mathematical Monthly, 46(5):281-283, 1939.

[2] Vasek Chvátal and Carsten Thomassen. Distances in orientations of graphs. Journal of Combinatorial Theory, Series B, 24(1):61-75, 1978.

[3] Fan RK Chung, Michael R Garey, and Robert E Tarjan. Strongly connected orientations of mixed multigraphs. Networks, 15(4):477-484, 1985.

[4] Fedor V Fomin, Martın Matamala, Erich Prisner, and Ivan Rapaport. At-free graphs: linear bounds for the oriented diameter. Discrete applied mathematics, 141(1-3):135-148, 2004.

[5] Fedor V Fomin, Martín Matamala, and Ivan Rapaport. Complexity of approximating the oriented diameter of chordal graphs. Journal of Graph Theory, 45(4):255-269, 2004.

[6] Nicole Eggemann and Steven D Noble. Minimizing the oriented diameter of a planar graph. Electronic Notes in Discrete Mathematics, 34:267-271, 2009.

[7] Sheng Bau and Peter Dankelmann. Diameter of orientations of graphs with given minimum degree. European Journal of Combinatorics, 49:126-133, 2015.

[8] Michel Surmacs. Improved bound on the oriented diameter of graphs with given minimum degree. European Journal of Combinatorics, 59:187-191, 2017.

[9] Paul Erdős, Janos Pach, Richard Pollack, and Zsolt Tuza. Radius, diameter, and minimum degree. Journal of Combinatorial Theory, Series B, 47(1):73-79, 1989.

[10] Peter Dankelmann and Michael Dorfling. Diameter and maximum degree in eulerian digraphs. Discrete Mathematics, 339(4):1355-1361, 2016. 
[11] Peter K Kwok, Qi Liu, and Douglas B West. Oriented diameter of graphs with diameter 3. J. Comb. Theory, Ser. B, 100(3):265-274, 2010.

[12] Yuefang Sun, Xueliang Li, Hengzhe Li, and Xiaolong Huang. Oriented diameter and rainbow connection number of a graph. Discrete Mathematics \& Theoretical Computer Science, 16, 2014. 